\title{
Anabases
}

ANABASES Traditions et réceptions de l'Antiquité

13 | 2011

Varia

\section{Alle origini dell'antropologia storica: Louis Gernet lettore di Hermann Usener}

Ilaria Sforza

\section{(2) OpenEdition}

Journals

Edizione digitale

URL: http://journals.openedition.org/anabases/1790

DOI: 10.4000/anabases. 1790

ISSN: 2256-9421

Editore

E.R.A.S.M.E.

\section{Edizione cartacea}

Data di pubblicazione: 1 marzo 2011

Paginazione: 131-156

ISSN: 1774-4296

\section{Notizia bibliografica digitale}

Ilaria Sforza, «Alle origini dell'antropologia storica: Louis Gernet lettore di Hermann Usener », Anabases [Online], 13 | 2011, Messo online il 01 mars 2014, consultato il 21 octobre 2019. URL : http:// journals.openedition.org/anabases/1790 ; DOI : 10.4000/anabases.1790

Questo documento è stato generato automaticamente il 21 ottobre 2019

(c) Anabases 


\title{
Alle origini dell'antropologia storica: Louis Gernet lettore di Hermann Usener
}

\author{
Ilaria Sforza
}

There is no natural phenomenon and no

phenomenon of

human life that is not capable of a mythical

interpretation,

and which does not call for such an

interpretation.

E. Cassirer, An Essay on Man:

An Introduction to a Philosophy of Human Culture,

New Haven 1944.

1 Nell'affrontare un tema che non appare esplicitamente trattato dalla critica degli ultimi anni, quello dell'influsso delle ricerche mitologiche e storico-religiose del filologo tedesco Hermann Usener (1834-1905) ${ }^{1}$ sull'ellenista e sociologo francese Louis Gernet $(1882-1962)^{2}$, sarà bene tener presente l'interlocuzione di Gernet, particolarmente intensa negli anni Quaranta del secolo scorso, con il padre della psicologia storica Ignace Meyerson (1888-1983) ${ }^{3}$.

2 In una lettera a Meyerson del 9 luglio 1942, Gernet annunciava l'elaborazione di un essai général d'analyse du mythe pour l'intelligence de la préhistoire sociale ${ }^{4}$. Lo stato di profondo coinvolgimento emotivo dello studioso francese, occupato nell'isolamento di Algeri, che si sarebbe protratto per altri sei anni, a condurre un'analisi mitologica sulla «preistoria sociale» della Grecia antica riflette l'importanza e le aspettative sul tema in questione ${ }^{5}$.

Un problema che tormentava Gernet, come si ricava dagli schemi allegati al fascicolo di appunti, databile tra il 1942 e il 1948, contrassegnato dalla lettera greca Y (Psychologie), la stessa con cui l'ellenista siglava i materiali destinati al saggio «La notion mythique de la valeur en Grèce» (1948), riguarda il diacronismo ${ }^{6}$ : 
«Diachronisme. Conditions désespérées en apparence. Mais d'autre part, richesse

des images peut-être instructive. Rôle des images.»

Come osserva Ernst Cassirer (1874-1945) nel secondo volume della Filosofia delle forme simboliche (Philosophie der symbolischen Formen, 1923-1929) dedicato al pensiero mitico (Das mythische Denken, 1925), la coscienza mitica si trova ancora, nelle sue fasi più antiche, in uno stadio di indifferenziazione; vi domina "un tempo assolutamente preistorico ${ }^{7}$ ». Di conseguenza, risulta problematica per Gernet la scelta del metodo da utilizzare nell'esame dei dati documentari di quella che egli definisce "préhistoire sociale». Se da un lato la diacronia in questa fase di proto-storia o di preistoria sociale pone un problema apparentemente irrisolvibile, dall'altro è la ricchezza delle immagini mitiche a fornire una chiave di lettura dei dati risolutiva. Tale ricchezza consentirà a Gernet di mettere a punto un nuovo metodo di lettura delle storie mitiche ${ }^{8}$ :

«Il n'y a pas de "méthode" pour l'analyser. Il faut lire des histoires, simplement. Mais des histoires supposent ou suggèrent certaines attitudes humaines: il convient d'y avoir égard si on veut lire comme il faut. Et une histoire en entraîne une autre : il y a des similitudes qu'il est bon de ne pas laisser échapper a priori par phobie du rapprochement arbitraire. Au fond, on ne demande qu'une permission, qui est d'admettre qu'une mythologie est une espèce de langage.»

Hermann Usener, in quella che è ancora oggi la sua opera più conosciuta, I nomi degli dèi (Götternamen, 1896), si proponeva di studiare «gli stadi dell'infanzia dei popoli» che sono la culla della religione popolare nonché della produzione delle forme mitiche. Le impressioni esterne che eccitano l'animo umano in questa età primitiva continuano a vivere nel patrimonio di miti del popolo. Ragion per cui, secondo Usener, «la materia della mitologia coincide completamente con la preistoria interiore o spirituale dei popoli storici che quelle mitologie hanno creato ${ }^{9} »$.

Il filosofo tedesco Ernst Cassirer, in Linguaggio e mito (Sprache und Mythos, 1925), dove elogia il metodo applicato da Usener allo studio del mito, osserva che non l'analisi dei vocaboli, come credeva l'eminente filologo, bensì l'indagine filosofica ed etimologica gli servono da strumento per indagare un problema più profondo e più vasto, l'«affiorare» dei nomi e delle rappresentazioni mitiche alla coscienza umana. Questo affiorare non è visto da Usener come un processo storico, ma come una struttura propria della coscienza mitica. Dall'analisi del singolo è possibile e necessario risalire alla legge ${ }^{10}$ :

«È soltanto immergendosi con abnegazione in queste tracce spirituali di un tempo trascorso, vale a dire mediante il lavoro filologico, che noi possiamo educarci a sentire quel che altri hanno sentito [...]. Osservazioni e confronti più approfonditi ci permetteranno di avanzare nella ricerca, così che ci si eleverà dal particolare al generale, dal fenomeno alla legge [...] Più si scava in profondità, più si è compensati da conoscenze universali.»

\section{Le ricerche di Gernet sulla preistoria sociale}

Gernet mostra di conoscere Le storie del diluvio (Die Sintfluthsagen, 1899) di Usener già ben prima della lettura risolutrice degli anni Quaranta ad Algeri. Le cita ad esempio nel saggio «Frairies antiques» (1928), a proposito dell'«ascesa» e della «discesa» simbolica del dio nelle feste antiche, ricordando il «Dionysos à la larnax et au vaisseau» di cui Usener si era occupato ne Le storie del diluvio ${ }^{11}$; esprime, tuttavia, una riserva: «Pour ce qui nous intéresse, la portée de ces transpositions mythiques se laisse mal définir ${ }^{12}$.» 
8 Eppure proprio nel milieu contadino Usener aveva individuato nella sua ultima opera, San Ticone (Der heilige Tychon, 1907), le radici di quel nesso mitico-rituale che si sostanzia nell'azione del bere il vino, la cui persistenza nella cultura cristiana è oggetto della sua disamina su Priapo-Ticone ${ }^{13}$. Non solo, nelle premesse al saggio sulle frairies antiche Gernet pone l'attenzione sul nesso mito-rito in riferimento al sacrificio nelle feste collettive della Grecia antica: «Dans la religion de la cité, les festins collectifs tiennent une large place. Dans les rites et dans les mythes, la cuisine est une grande affaire ${ }^{14} »$, facendo riferimento al ruolo centrale di Dioniso, dio della vegetazione e della vite ${ }^{15}$.

9 Poco oltre, nello stesso saggio, compare la definizione di «préhistoire sociale», che sarà alla base dell'indagine, progettata e mai portata a termine, sulla leggenda greca. Qui la preistoria sociale è definita «sfondo primitivo ${ }^{16} \%$ :

«De ce fonds primitif de société, de morale, de religion, il n'est pas question de montrer tout ce qui a pu sortir; ce serait refaire d'une certaine manière la préhistoire - une préhistoire abstraite et conjecturale.»

10 Trapela da queste considerazioni quella difficoltà nel ricostruire il diacronismo della mentalità primitiva che Gernet non riuscirà a risolvere se non ricorrendo al nesso useneriano, ripreso sul piano filosofico da Cassirer, tra linguaggio e mito ${ }^{17}$.

11 In " Frairies antiques », che risale a una fase ancora iniziale della sua riflessione sulla leggenda greca, Gernet adotta un criterio che gli consente di attenersi il più possibile alle fonti: egli parte nel suo esame dai termini associati alle feste contadine, considerandoli «l'expression d'une pensée singulièrement antique, tenace et continue ${ }^{18}$ $»$.

12 Nelle conclusioni lo studioso francese si richiama a Mutter Erde (1905) ${ }^{19}$ di Albrecht Dieterich (1866-1908), cognato e collaboratore di Usener, in merito alla partecipazione della natura alle azioni rituali degli uomini durante le feste campestri e allo stretto legame tra matrimoni collettivi e presenza dei morti che assicurano il rinnovamento della natura e favoriscono la produzione dei frutti della terra. Nell'esame dei termini

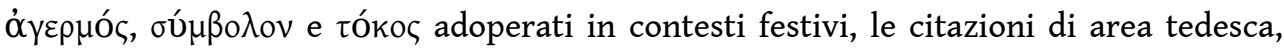
segnatamente Dieterich e Usener, si alternano a quelle di esponenti della scuola sociologica francese, come Gustave Glotz (1862-1935) e soprattutto Marcel Mauss $(1872-1950)^{20}$.

13 La speciale considerazione che Gernet aveva per i due studiosi di area tedesca menzionati, la si desume del resto anche dalla sua recensione a Der Glaube der Hellenen (1931-1932) di Ulrich von Wilamowitz-Möllendorff (1848-1931) ${ }^{21}$, apparsa nel 193422. Qui Gernet afferma che «dans le domaine propre de l'"histoire des religions", on ne saurait le [Wilamowitz] mettre en parallèle avec un Usener ou un Dieterich, voire avec un Rhode $^{23}$ ». Tale giudizio è motivato a partire dallo scarso interesse di Wilamowitz per l'«histoire de l'homme»; una prova ne è l'impazienza del filologo tedesco di «sortir d'un passé un peu trop nébuleux et peut-être un peu trop barbare ${ }^{24}$ ». L'atteggiamento di Wilamowitz è agli antipodi rispetto alle ricerche di Gernet sulla leggenda greca indirizzate per molti anni proprio a quel passato nebuloso che poco interessava al filologo tedesco ${ }^{25}$. Del resto, conclude Gernet, «Il faut le prendre comme il est. Il est essentiellement un philologue, au sens le plus large et le plus élevé du mot ${ }^{26} \%$. Non sarà necessario richiamare qui le note divergenze tra Wilamowitz e Usener, che si andarono accentuando man mano che gli interessi di quest'ultimo si rivolgevano alla storia delle religioni, per spiegare la diversa considerazione di Gernet nei confronti dei due studiosi tedeschi entrambi formatisi all'università di Bonn ${ }^{27}$. 
14 Ne «La cité future et le pays des morts» (1933), «la più dettagliata analisi di sistemi di immaginazioni mitiche condotta da Gernet ${ }^{28}$ », lo studioso rinvia a Le storie del diluvio (Die Sintfluthsagen) per la connessione tra le rappresentazioni del paese degli dèi, del paese dei Morti e del paese dei frutti meravigliosi, toccando solo marginalmente il tema del «tesoro celeste» che approfondirà ne «La notion mythique de la valeur en Grèce ${ }^{29}$ »:

«Il n'y a pas lieu ici - je me contente de renvoyer, notamment, à Usener - de marquer la parenté intime, ou plutôt l'identité profonde, entre les représentations du pays des Dieux, du pays des Morts, du pays des fruits merveilleux - et aussi, naturellement, de l'âge d'or.»

Gernet persegue, anche in questo saggio, la ricerca di un sostrato più antico del mito attraverso le tracce, nei romanzi utopici fioriti nell'età di Alessandro, di miti risalenti a Esiodo - il mito delle razze -, se non a Omero - il paese dei Cimmeri, dei Lotofagi ecc. e forse riconducibili a un fondo greco-orientale, a una "tradition étrangère et antérieure aux Hellènes» come era stato già ipotizzato, tra gli altri, dal filologo svedese Martin P. Nilsson (1874-1967) ${ }^{30}$.

Ancora più significativo è l'influsso di Usener sul saggio «Dolon le loup» (1936), dove Gernet sembra, in un primo tempo, prendere le distanze dall'approccio del filologo tedesco al mito, definendo «un peu aériennes» le sue ricostruzioni mitologiche, pure riconoscendogli un'intuizione importante: «C'est que l'être mythique représenté par le loup est tour à tour vainqueur et vaincu ${ }^{31} . »$

17 A ben vedere, il particolare già omerico che Dolone si riveste della pelle di un lupo grigio (Il. X 334), opportunamente sottolineato da Usener come tratto «rivelatore» nella sua indagine sulle origini religiose dell'epopea, costituisce il punto di partenza della disamina di Gernet. Questi, rifacendosi alle sue ricerche precedenti sulle feste religiose nelle frairies antiche, si sofferma sull'aspetto rituale che il mito di Dolone presupporrebbe tanto nel Reso pseudo-euripideo, quanto nella Dolonia omerica. La divergenza tra Gernet e Usener è da ravvisare piuttosto sul versante della ricostruzione etimologica, che rivela talvolta la fragilità dell'impianto argomentativo dello storico delle religioni tedesco ${ }^{32}$.

L'obiettivo di individuare all'interno di una «histoire legendaire» dei temi rituali, seppure multipli e mescolati tra loro, traspare in uno snodo essenziale dell'argomentazione ${ }^{33}$ :

«On ne s'attend pas à pouvoir reconnaître par transparence, dans un récit incorporé à une histoire légendaire, une série complète et ordonnée de thèmes rituels. Elles sont nécessairement multiples et entremêlées, les images qui ont été retenues par une mémoire collective passablement capricieuse.»

19 Gernet torna ancora una volta sul concetto di «preistoria sociale» esprimendo la convinzione che all'origine di alcuni tratti mitici del racconto trattato nel Reso vi siano «des rites et notions préhistoriques que l'épopée avait complètement transposés» e conclude $^{34}$ :

«De ce principe d'interprétation, je ne crois pas devoir me justifier à nouveau ; j'en répéterai volontiers ce qu'en disait Pierre Roussel dans une occasion analogue : je ne sache pas qu'il soit périmé.»

Più in generale, è possibile ravvisare in "Dolon le loup» il tentativo di cogliere quel nesso tra mito e rito che era stato l'oggetto delle ricerche storico-religiose dell'ultimo Usener, dal cui contributo «Heilige Handlung» (1901) Gernet era partito per le sue considerazioni sul personaggio di Dolone. 
21 Anche il contributo sulle «Vieilles légendes de Grèce» (1936) appare orientato, fin dalle premesse, in direzione di quel saggio generale di analisi del mito per l'intelligenza della preistoria sociale annunciato a Meyerson nella lettera del $1942^{35}$ :

«La légende grecque a parfois gardé le souvenir de pratiques sociales très anciennes, et il arrive même que les récits se sont organisés autour de cette donnée archaïque, plus ou moins bien retenue, plus ou moins bien comprise.»

Ben diverso è, in ogni caso, il tenore di questa ricapitolazione di storie leggendarie, che «ne sont peut-être pas celles qui s'y prêteraient le mieux», - la pratica del fosterage, la caccia al cinghiale Calidonio, Erifile la donna fatale, Filomela o il matrimonio forzato, il demone-lupo di Temesa - rispetto al saggio sulla nozione mitica del valore.

Le leggende prese in esame da Gernet in questo contributo non sono prive di spunti interessanti, ma appaiono come una sorta di rivisitazione dei temi mitici che lo avevano occupato negli ultimi anni; manca una visione d'insieme, un metodo in grado di unificare i dati forniti da ciascuna storia, mancano infine quelle connessioni e associazioni risolutive che emergono dagli appunti databili tra il 1942 e il 1948.

\section{La nozione mitica del valore: premesse di metodo}

L'influsso della psicologia storica di Ignace Meyerson sul contributo più significativo di Gernet relativo al mito è percepibile fin dall'esordio ${ }^{36}$ :

«Il y a des fonctions mentales, comme celles du droit et de l'économie, dont pour un peu on oublierait qu'elles en sont : c'est qu'elles s'accomplissent dans nos sociétés suivant un mécanisme dont l'homme lui-même paraitrait absent.»

Il diritto e l'economia sono dette da Gernet in questo incipit «fonctions mentales» con un chiaro riferimento alla tesi di Meyerson, apparsa nello stesso anno, Les fonctions psychologiques et les oeuvres ${ }^{37}$. Gernet in una lettera del 6 febbraio 1948 aveva rivolto all'amico una bonaria critica proprio in merito al termine fonction ${ }^{38}$ :

«En tant que lecteur "idiot", il y a une petite critique que je vous adresserais : je sais bien que le terme fonction est d'usage courant, mais je ne le vois pas défini au cours du livre, et je me demande s'il n'aurait pas été utile de le faire en forme.»

Innanzitutto la destinazione del contributo di Gernet, il Journal de Psychologie Normale et Pathologique, diretto al tempo dallo stesso Meyerson, influisce sulla scelta del lessico che l'ellenista francese adopera nel tentativo di «tradurre» le sue riflessioni sul mito e la società greca delle origini in termini psicologici. Sulla stessa rivista era apparso, nel 1933, «Le langage et la construction du monde des objets» di Ernst Cassirer, in cui il filosofo riprendeva alcune delle idee espresse in Linguaggio e mito (Sprache und Mythos) e, in modo più esteso, nella Filosofia delle forme simboliche (Philosophie der symbolischen Formen), applicandole nello specifico all'apprendimento del linguaggio e alla costruzione del mondo degli oggetti da parte del bambino ${ }^{39}$.

Ma l'indagine di Gernet, che pure si muove sul fragile e fecondo terreno di scambi tra le diverse discipline offerto dalla psicologia storica, è imperniata su una «nozione» piuttosto che su una «funzione». La distanza tra questi due termini sta tutta nel carattere più coraggiosamente definitorio del primo rispetto a quello volutamente relativo e variabile del secondo ${ }^{40}$. La nozione del valore, poi, è una nozione mitica prima ancora che psicologica, dunque implica quello scavo nel passato che da lungo tempo aveva occupato Gernet. L'incontro tra i due ambiti distinti, ma per molti aspetti affini, 
della psicologia e della mitologia avviene proprio a partire dalla definizione di notion globale $^{41}$ :

«La notion de valeur qu'on y rencontre reste une notion globale: elle participe de ce qui est objet de respect, voire de crainte révérentielle, principe d'intérêts, d'attachement ou d'orgueil, motif de cette admiration dont Descartes faisait la première "passion primitive".»

La nozione mitica del valore è, in ultima istanza, una nozione simbolica, concepita da una mentalità «primitiva», le cui tracce sono ravvisabili ancora in età storica ${ }^{42}$ :

«L'expérience n'a sans doute que plus d'intérêt si elle porte sur le stade qui touche déjà, chronologiquement, à l'âge "positif" de la valeur et où ne s'en prolongent pas moins des formes psychologiques qui relèvent d'une très vieille tradition.»

L'origine della moneta è vista da Gernet come il passaggio dal «simbolo» al «segno» secondo la distinzione che tra questi termini aveva postulato Meyerson ${ }^{43}$. Un simile passaggio è ravvisabile nel diritto e dal momento che i giochi nella Grecia antica preludono ai gesti regolamentati del diritto arcaico, i premi dei giochi sono da intendersi come «segni premonetari». Nell'uso linguistico la nozione del valore, in questo stadio molto antico che precede il concetto più astratto di valore economico, è espressa dal termine agalma, «oggetto prezioso», già in Omero ${ }^{44}$.

Il secondo paragrafo, più interessante per il confronto con Usener, introduce un altro piano d'indagine, quello della représentation mythique ${ }^{45}$. Tale représentation mythique, intesa qui come sinonimo di légende, richiama le mythische Vorstellungen indagate da Usener nel sesto capitolo de Le storie del diluvio (Die Sintfluthsagen), e così interpretate dal filosofo Ernst Cassirer ${ }^{46}$ :

«Esse non sono la sublimazione di un mondo di realtà già bello e pronto, non sono semplici figurazioni della fantasia, che si distacchino dalla solida realtà empiricamente esistente degli oggetti e si elevino come una lieve nebbia al di sopra di essi, bensì esse per la coscienza primitiva rappresentano la totalità del reale.»

31 Nell'introdurre il piano delle rappresentazioni mitiche, Gernet dice che è possibile osservare, a partire da esso, «l'activité mentale par quoi la valeur se constitue, c'est-àdire s'objective ${ }^{47}$ », richiamandosi significativamente alle riflessioni sull'oggettivazione nel pensiero mitico contenute nel primo capitolo della tesi di Meyerson, intitolato proprio «L'objectivation ${ }^{48}$ ». L'influsso avuto sul fondatore della psicologia storica dal filosofo tedesco Cassirer, che aveva trattato a più riprese questo tema, può essere oggi difficilmente messo in dubbio ${ }^{49}$.

Se davvero l'equilibrio generale delle citazioni nella tesi di Ignace Meyerson è istruttivo, come afferma Jean-Pierre Vernant nella sua prefazione alla traduzione italiana dell'opera, non passerà inosservato il numero consistente delle citazioni che vi si trovano di Ernst Cassirer (9), di poco superiore al numero di quelle del grecista Louis Gernet (7), amico e maestro di Meyerson ${ }^{50}$. Cassirer è ricordato da Meyerson soprattutto nel primo capitolo della sua tesi.

Una prima citazione estesa di Cassirer fa riferimento all'articolo apparso nel 1933 sul Journal de psychologie ${ }^{51}$ :

«Le langage [...] est, en un sens, le médiateur par excellence, l'instrument le plus important et le plus précieux pour la conquête et pour la construction d'un vrai monde d'objets.»

Nel paragrafo intitolato "L'objectivation dans la pensée mythique», Meyerson torna a citare Cassirer, e in particolare il secondo volume della Filosofia delle forme simboliche (Philosophie der symbolischen Formen) dedicato al pensiero mitico ${ }^{52}$. Come si è avuto modo 
di osservare per le citazioni di Usener da parte di Gernet nei saggi precedenti al 1948, anche nell'opera di Meyerson la ripresa delle idee di Cassirer si integra con i riferimenti ad autori più vicini a lui per provenienza e interessi culturali, tra questi soprattutto il sinologo Marcel Granet, citato ben 12 volte e Marcel Mauss, a cui spetta la palma assoluta (18 citazioni, il doppio di Cassirer!). Il riferimento di Cassier al contributo dei francesi Henri Hubert e Marcel Mauss, «Esquisse d'une théorie générale de la magie» (1902/1903), suggerisce la reciprocità dell'interlocuzione ${ }^{53}$.

Dell'«onnipotenza del nome», del resto, Cassirer aveva più diffusamente parlato in Linguaggio e mito (Sprache und Mythos), dove assegnava a Usener un posto di tutto rispetto accanto a Platone, a partire dall'interesse, comune ad entrambi, per i nomi degli dèi. Secondo Cassirer ${ }^{54}$ :

«Che il nome e l'essenza siano tra loro in una connessione intrinsecamente necessaria, e che il nome non soltanto designi l'essenza ma che esso sia l'essenza medesima, e che la forza dell'essenza medesima stia racchiusa in esso, tutto ciò rientra nei presupposti fondamentali della stessa intuizione mitica.»

Ben altro giudizio è espresso da Cassirer nei riguardi di altri studiosi del XIX secolo le cui teorie in merito alla relazione tra linguaggio e mito «ricordano ancora in modo inconfondibile gli antichi metodi della sofistica greca ${ }^{55}$ ». Per Max Müller, ad esempio, che concepiva il mondo mitico come semplice mondo dell'apparenza, il mito, generato da una sorta di deficienza dello spirito, non sarebbe altro che una «malattia» condizionata dal linguaggio ${ }^{56}$.

Nella sua disamina su linguaggio e mito, Cassirer aveva fatto suoi i presupposti dell'indagine condotta da Usener sui nomi degli dèi, riconoscendole un'intenzionalità filosofica, espressa dal sottotitolo Saggio di una teoria della formazione dei concetti religiosi (Versuch einer Lehre von der religiösen Begriffsbildung). Usener si era proposto di tracciare non una storia delle figure degli dèi, bensì una storia delle rappresentazioni che di essi si ebbero. Tali rappresentazioni hanno una propria legge intrinseca, che la mitologia

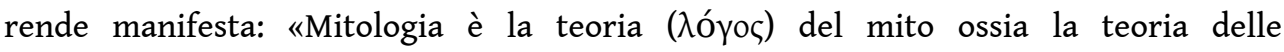
rappresentazioni religiose ${ }^{57} . »$

Ciò che il filosofo apprezza soprattutto dell'indagine condotta da Usener è che ${ }^{58}$ :

«Questa indagine cerca di penetrare fino ad un punto in cui l'uno e l'altro, così il dio come il suo nome, cominciano ad affiorare nella coscienza. Questo affiorare [...] si cerca di comprenderlo in base puramente alla struttura fondamentale della coscienza linguistica e mitica, in base ad una legge generale della formazione concettuale linguistica e religiosa. Qui dunque noi non siamo più nel campo della storia, ma in quello della fenomenologia dello spirito.»

L'esempio scelto da Gernet per esemplificare la nozione mitica del valore in Grecia, vale a dire quegli oggetti preziosi, agalmata, che figurano nei racconti mitici e che ne sono in qualche modo i protagonisti, "car ils ne laissent pas d'y être animés d'un pouvoir propre» rispecchia il tipo di immaginazione che contraddistingue, secondo Usener, gli «stadi dell'infanzia dei popolis ${ }^{59}$ :

«Sono gli stadi dell'infanzia dei popoli quelli sui quali si edifica la religione popolare e si producono forme mitiche; tutto ciò che in questa fase dell'esistenza eccita l'animo del popolo, il mondo esterno nella sua totalità, i primi cenni di coscienza [...] tutto ciò continua a vivere come riflesso nel patrimonio di miti del popolo, ragion per cui presso quegli uomini ogni evento nuovo e sconosciuto appare innanzitutto come un'essenza divina. La materia della mitologia coincide dunque completamente con la preistoria interiore o spirituale dei popoli storici che quelle mitologie hanno creato.» 
40 È dunque il passaggio dallo studio della leggenda come forma di preistoria sociale «astratta e congetturale» allo studio della «preistoria interiore e spirituale dei popoli storici» come la intendeva Usener che consente a Gernet il superamento di quell'impasse che gli aveva impedito di condurre a termine il progettato saggio sulla leggenda greca. La soluzione da lui trovata nel 1948 consiste nell'analisi di una nozione «globale», la nozione di valore, prodotto di una società preistorica e premonetaria, che precede di poco l'avvento del pensiero razionale.

41 Cassirer, interpretando il pensiero di Usener, aveva così descritto il modo di procedere della coscienza mitica ${ }^{60}$ :

"Questa coscienza è e vive nell'impressione immediata, alla quale si abbandona senza "metterla a confronto" con altro. Per essa l'impressione non è qualcosa di relativo, ma di assoluto; non è "in virtù" di altro e non dipende da altro, ma si afferma e si convalida con la semplice intensità della sua esistenza, con la violenza con cui si impone alla coscienza.»

42 Non occorre postulare una mediazione di Cassirer - che non si può tuttavia escludere -, per affermare che la concezione del mito come prodotto della rappresentazione inconscia, così carica di conseguenze per la definizione di un metodo di lettura delle storie mitiche, Gernet l'aveva ricavata proprio da Usener.

Tra i numerosi richiami allo studioso tedesco, nel fascicolo di appunti contrassegnato con l'emblematica lettera $\Psi$, risalta un'intera pagina di annotazioni da «Mythologie» (1904). La riportiamo di seguito con le sottolineature e i corsivi originali ${ }^{61}$ :

«Symbole et mythe renvoient à la même source: Beide entspringen denselben Vorgängen unbewussten Vorstellens, dont l'importance a d'abord été reconnue par Vico.

Les deux processus principaux de la représentation mythique = Beseelung (Personifikation) et Verbildlichung (Metapher)... Aber auch Sprache und Dichtung strecken ihre Wurzeln in diesen geheimnisvollen Grund: constitution mentale et forme de mouvement qui a son antithèse dans la pensée rationnelle et la science. Diese Vorgänge verlaufen unwillkürlich und unbewusst wie Wirkungen physiologischer Reize, und stehen darum, mit Ausnahme der Analogetik, ausserhalb aller Denkgesetze, tout en possédant pour l'esprit certitude et réalité immédiates. Images qui se détachent conformément à la mentalité collective pour être finalement rejetées aux mythes par la science.

Pour la représentation mythique Ding et Bild coïncident, pour la pensée rationnelle, ils se présentent consciemment comme distincts zu Vergleichung und Gleichnis.»

L'influsso avuto da Usener sull'elaborazione di un nuovo metodo di interpretazione del mito da parte di Gernet non si limita alla matrice inconscia della rappresentazione mitica, ma riguarda anche il nesso linguaggio e mito. Nel medesimo fascicolo $Y$ un intero paragrafo è intitolato «Mythologie et langage. Point de vue langue». Qui Gernet prende spunto ancora una volta da Usener, che lamentava nel suo saggio «Mythologie» la mancanza di un carattere «scientifico» dell'indagine mitologica ${ }^{62}$ :

«Ce qui n'est pas interdit, en revanche, c'est la réflexion sur les procédés ou démarches qui semblent avoir abouti ça et là, qui semblent, sur tel ou tel point, fournir au moins pour autant cette intelligibilité, autrement dit ce caractère "scientifique", que requérait un savant illustre il y a quarante ans et qu'il se plaignait de ne pas trouver dans les exposés d'ensemble.»

Partendo da Usener, Gernet approda a un nuovo tipo di indagine mitologica, che non è necessariamente storica, basata sulla considerazione che «un mythe n'est pas isolé: il s'articule avec d'autres; il en suppose ou en évoque d'autres ${ }^{63}$ ». La suggestione prodotta 
da questi rapprochements è così forte che persino il confronto con i dati forniti dalla linguistica gli appare certamente utile, ma non primario.

Poco oltre, Gernet giunge alla formulazione del concetto di polyvalence des images, che egli definisce un phénomène de mémoire sociale $e^{64}$. La trasmissione delle immagini non è un processo continuo; solo alcune di esse vengono selezionate per essere riutilizzate anche in seguito in contesti nuovi. L'immagine, proprio come la parola nella linguistica di Ferdinand de Saussure (1857-1913), a cui il grecista fa riferimento, può cambiare radicalmente di significato se inserita in un contesto nuovo. Se dunque da un lato stabilire una cronologia, ovvero «ordinare» le immagini del mito, rimane un'impresa disperata, dall'altro, proprio la conservazione di alcune di esse a discapito di altre, fa presumere che tali immagini avessero un significato particolare ${ }^{65}$.

Di qui la rinuncia a stabilire una cronologia, in favore dell'osservazione di due fattori, che saranno il criterio guida del saggio sulla nozione mitica del valore: le associazioni e le connessioni ${ }^{66}$. Sappiamo, a partire da queste pagine di appunti, che il termine connexion corrisponde in Gernet a Vieldeutigkeit, con il quale sintetizza due caratteristiche attribuite da Usener alle rappresentazioni mitiche, la varietà (Vielfaltigkeit) e la polisemia (Mehrdeutigkeit): «Ce que Usener nommait Vieldeutigkeit, nous le comprenons comme connexion ${ }^{67}$.» Accanto alle connessioni e insieme ad esse, le associazioni «en vertu desquelles un épisode, un motif ou une image évoquent une série similaire» aiutano, in un certo senso, a comprendere ${ }^{68}$.

Nel saggio del 1948 Gernet torna sul paragone tra mitologia e linguaggio avvalendosi della distinzione tra significante e significato operata da Ferdinand de Saussure ${ }^{69}$. Questa volta il riferimento è più specifico: i rapporti sintagmatici e associativi che regolano il funzionamento dei significanti di una lingua trovano corrispondenza nelle connessioni e associazioni che intercorrono tra le rappresentazioni mitiche. Gernet adatta qui la terminologia saussuriana al metodo applicato da Usener allo studio delle rappresentazioni mitiche. Nel paragrafo sulle «solidarités syntagmatiques» a cui Usener fa riferimento, de Saussure ne dava la seguente definizione: «Presque toutes les unités de la langue dépendent soit de ce qui les entoure sur la chaîne parlée, soit des parties successives dont elles se composent elles-mêmes ${ }^{70}$.» Non solo, rapporti associativi e rapporti sintagmatici si influenzano a vicenda: «Le tout vaut par ses parties, les parties valent aussi en vertu de leur place dans le tout, et voilà pourquoi le rapport syntagmatique de la partie au tout est aussi important que celui des parties entre elles.» Infatti, «la coordination dans l'espace contribue à créer des coordinations associatives, et celles-ci à leur tour sont nécessaires pour l'analyse des parties du syntagme ${ }^{71} »$. De Saussure conclude affermando che «l'idée appelle, non une forme, mais tout un système latent, grâce auquel on obtient les oppositions nécessaires à la constitution du signe ${ }^{72} »$.

Se si applica, seguendo Gernet, questa teoria linguistica alle rappresentazioni della mitologia, si dovrà tenere conto allo stesso tempo delle connessioni che esistono all'interno di elementi e momenti di una storia data (rapporti sintagmatici), e delle associazioni in virtù delle quali un motivo o un'immagine evocano una serie similare (rapporti associativi). Questi due ordini di rapporti, come aveva suggerito de Saussure, si influenzano a vicenda. 


\section{I temi mitici e le immagini}

50 La prima storia mitica presa in esame da Gernet, il tripode dei Sette Saggi, riguarda un oggetto che, se da un lato figura spesso come premio di una gara, dall'altro, acquista un valore particolare in quanto $s a \mathrm{cro}^{73}$. Rientra cioè in quella categoria la cui ambiguità linguistica e semantica era stata sottolineata da Cassirer: il sacro è allo stesso tempo una categoria positiva e negativa, ciò che è consacrato è sia venerato che intoccabile, quasi maledetto. Secondo il filosofo tedesco, infatti, l'unicità degli oggetti mitici, che si impongono con forza e immediatezza alla coscienza primitiva, coesiste con un principio universale comune: una tensione verso la «trascendenza» che riunisce e accomuna tutti i contenuti della coscienza mitica e di quella religiosa; questa compenetrazione conferisce al contenuto mitico-religioso il suo carattere distintivo, il carattere del «sacro» ${ }^{74}$.

51 È quindi possibile, secondo Gernet, "presentire» una rappresentazione mitica che si avvale della caratteristica, topica per questo genere di oggetti di artigianato prezioso, di essere gettati e quindi ripescati dal mare. Il confronto è con l'oggetto che nasconde l'eroe bambino e lo restituisce alla luce, l'arca nel mito di Perseo. Il riferimento a Le storie del diluvio (Die Sintfluthsagen), dove Usener si era occupato a lungo di questo mito e dei significati simbolici dell'arca, è d'obbligo ${ }^{75}$. Il carattere nefasto dell'oggetto sacro si manifesta nel caso del tripode a partire dal momento in cui Elena, gettandolo in mare, predice che esso costituirà il premio di una contesa. L'oggetto appare dotato di un'efficacia misteriosa: esso esercita, nel senso proprio del termine, un influsso nefasto. Questo tema, inutile rispetto alla storia, è stato da questa ostinatamente conservato, dunque esso appartiene alla nozione stessa dell'oggetto prezioso ${ }^{76}$. Gernet osserva, inoltre, che le varianti del mito che vedono la sostituzione del tripode con la coppa non presentano il particolare dell'immersione e poi del ritrovamento in mare; ne ricava un particolare nesso tra il simbolismo del tripode e un attributo essenziale della nozione mitica del valore. Le conclusioni di Gernet su questo primo racconto mitico offrono una conferma di quanto da lui postulato nelle premesse ${ }^{77}$ :

«Les symbolismes d'une même image ont beau avoir - pour nous - des directions

différentes : dans la représentation mythique, ils adhèrent entre eux.»

Anche nel secondo esempio, la collana di Erifile, Gernet vuole individuare le connessioni interne al racconto. Nelle conclusioni, adoperando l'espressione série légendaire si richiama espressamente alle "associazioni» saussuriane. Lo studioso osserva come il tema mitico della «forza obbligante» del dono, nello specifico della collana di Erifile, sia passato ormai in secondo piano nel mito, eppure vi persista: la représentation de l'objet de valeur non potrebbe farne a meno.

Ma osserva: «Il a d'autres aspects, aussi bien: d'autres formes de l'imagination se définissent dans certaines séries légendaires ${ }^{78}$.» Dietro il singolo racconto mitico si apre uno sfondo, paragonabile al contesto in linguistica, in grado di suggerire tutta una serie di associazioni. Questo sfondo, costituito da innumerevoli altri racconti che presentano analogie con quello considerato, è indispensabile per comprendere le implicazioni che quel racconto, o anche un solo elemento di esso, comporta. Come in un gioco di scatole cinesi, «une histoire en entraîne une autre», aveva detto Gernet all'inizio, «il y a des similitudes qu'il est bon de ne pas laisser échapper a priori par phobie du rapprochement arbitraire ${ }^{79} »$. 

Policrate è, come nel caso del tripode dei Sette Saggi, il fatto che l'oggetto prezioso venga gettato in mare, in questo caso dal suo stesso proprietario che tenta così di disfarsene, ma «la richesse, objet de respect religieux, peut-elle être détruite ?»: è questo il quesito con cui si apre la terza disamina. La presenza del medesimo gesto quello di gettare l'anello in mare - nel racconto mitico che ha come protagonista Teseo suggerisce a Gernet che si tratti di un gesto rituale ${ }^{80}$. È appena necessario un secondo confronto, quello con la storia dell'anello di Gige, dotato di poteri magici, per ricavare il significato simbolico dell'oggetto: l'anello è lo strumento della conquista del potere regale. Quest'ultimo esempio leggendario implica un nuovo tema, squisitamente useneriano, quello del «tesoro», poiché l'anello di Gige viene ritrovato in un sotterraneo, dimensione analoga alle profondità marine, in cui anche altri anelli preziosi sono celati nei rispettivi racconti. Attraverso una serie di confronti, che portano sul tema dell'ordalia, Gernet ricava il significato della pratica rituale a cui il racconto di Policrate attribuisce un senso di prova di regalità: «La consécration totale, par immersion, d'un objet précieux ${ }^{81} . »$

Il gesto dell'immersione di un oggetto prezioso in mare assume pertanto il valore di un tentativo di distruzione dell'oggetto medesimo, simbolo di ricchezza, e sebbene tale «sacrificio» distruttivo non abbia sempre un destinatario, esso ha in tutti i casi una direzione: è ciò che conta nel pensiero mitico. Si tratta di uno "schème imaginatif», che persiste in altri racconti mitici relativi ad agalmata anche in assenza degli elementi originari del racconto ${ }^{82}$. Ancora una volta Gernet, nell'interpretare il valore simbolico dei gesti, ai quali riconosce un carattere rituale, svolge la sua analisi su di un piano mitico, prescindendo dall'aspetto puramente narrativo, e in qualche caso storico, del racconto.

Nel paragrafo conclusivo della parte dedicata all'anello di Policrate, Gernet si sofferma ad indagare la proprietà degli agalmata di mediatori tra la sfera umana e quella divina, cui molto spesso allude il fondale marino, significativamente popolato di Nereidi nel racconto di Enalo, personaggio emblematico già solo per il suo nome. Dalla sfera subacquea nella quale questi eroi si immergono per poi tornare indietro, provengono gli oggetti preziosi, come la coppa d'oro nel racconto di Enalo, che partecipano di una natura divina ${ }^{83}$ :

«Comme d'autres objets mythiques... les objets de prix, symboles coutumiers de richesse, sont en relation nécessaire avec cet autre monde que postule la pensée religieuse : tour à tour ils y descendent et ils en proviennent.»

57 La quarta parte del saggio, dedicata ai racconti relativi al vello d'oro, appare la più complessa e ricca nella sua articolazione interna. Dopo avere esaminato storie più $o$ meno recenti o parti di leggende che esemplificano il modo di procedere dell'immaginazione mitica in una fase anteriore all'avvento del pensiero positivo, Gernet dichiara di volere risalire $\mathrm{a}^{84}$ :

«des formes plus anciennes, autrement étoffées et où les conceptions éparses qu'on a relevées chemin faisant se trouveraient accordées à la notion générale, mais plus profondément mythique, de richesse.»

Quale sia stato l'influsso esercitato da Usener su Gernet sotto questo particolare aspetto della sua ricerca lo abbiamo indicato in precedenza ${ }^{85}$. Basti richiamare qui le considerazioni condotte dal filologo tedesco nel sesto capitolo de Le storie del diluvio 
(Die Sintfluthsagen), a cui è affidata la chiave di lettura metodologica dell'intero saggio $^{86}$ :

«Sarebbe davvero difficile, se non impossibile, sbrogliare il tessuto narrativo, a cui hanno lavorato tante generazioni di poeti, logografi e mitografi con l'intento di sistematizzarlo, se non fossero presenti in molti casi delle forme mitiche più semplici, più antiche e non contaminate dal rimpasto che dei motivi fa la poesia, forme che si prestano al confronto e permettono di riconoscere il nocciolo del racconto mitico sviluppato o per meglio dire avviluppato dalla poesia.» una stessa rappresentazione, concetto espresso da Usener sotto il nome di Vielfaltigkeit. Di tutti questi significati «partecipa» l'idea di agalma. Nonostante il concetto di efficacia «regale» associato all'immagine mitica del vello d'oro appaia multiplo e ricco di ramificazioni quasi infinite, Gernet continua a perseguire, in uno sfondo preistorico, quella specie di unità che lascia intravvedere talvolta la plasticità stessa del simbolo. $\mathrm{E}$ questa unità, che traspare nell'associazione tra l'oro e il Sole, lo porta progressivamente verso il complesso di miti che potremmo definire, con Usener, del «tesoro celeste». Il fatto che l'oro costituisca il tramite del passaggio dall'oggetto 
simbolico del mito all'oggetto concreto, strumento della regalità, come lo scettro, comporta la messa a fuoco della nozione di valore ${ }^{90}$ :

«De la notion idéale de l'autre monde il y a comme une projection sur le plan de

l'humain : le trésor est une réalité sociale.»

Dietro alla realtà sociale, e indissolubile da questa, si staglia la realtà mitica: sono due facce della stessa medaglia. L'oro, in quanto mediatore di vita attraverso il suo valore simbolico, conferisce animatezza a oggetti inanimati e, allo stesso tempo, fa da tramite tra il mondo divino da cui l'oggetto proviene e il mondo umano, dove la nozione di valore si concretizza. La connessione tra la leggenda del vello d'oro e il tema useneriano del tesoro celeste ripreso da Gernet, trova conferma nel fatto che il vello d'oro è nascosto da Atreo in un'arca. Il tesoro regale viene in genere custodito in un luogo sotterraneo ed è sorvegliato da un re-custode.

Nel quinto paragrafo, riprendendo il filo delle sue riflessioni sulla nozione mitica del valore, Gernet offre una precisazione. È significativo che anche in questo snodo fondamentale del suo ragionamento, Gernet si richiami a Usener, e in special modo al saggio sulla mitologia del 1904, il cui valore di testamento metodologico non richiede qui alcun commento ${ }^{91}$ :

«Si une notion ancienne de la valeur se trouve illustrée par la tradition légendaire, il y a une bonne raison à cela: elle est mythique elle-même quant au mode de pensée. Ce qui signifie d'abord que des fonctions différentes [...] y sont plus ou moins confondues: elle tend à être totale [...]. Il n'est pas interdit pour autant d'y reconnaître une espèce de pensée (sur cette fonction de la mythologie, cf. H. Usener, "Mythologie"), puisqu'on y a reconnu, au fur et à mesure, des directions.»

Usener aveva affermato in «Mythologie»: «Solo se inclusa nella storia della religione di un popolo la sua mitologia è in grado di trovare una trattazione completa ${ }^{92} . »$ Ma il perimetro delle rappresentazioni religiose, oggetto di indagine da parte della mitologia, è incomparabilmente vasto. Tali rappresentazioni derivano dalle più profonde impressioni dell'animo umano; «si potrebbe dire che l'uomo primitivo percepisse solo religiosamente ${ }^{93}{ }^{\prime}$. Questo modo di percepire non è proprio solo dell'umanità primitiva; esso ha lasciato un segno profondo nelle credenze divine e nel mito. La mitologia ha il compito, secondo Usener, di invadere il campo della storia delle religioni fino a «germogliare» anche nella storia della cultura, ovvero nella storia dello spirito dell'umanità. $\mathrm{E}$ «lo spirito umano procede in modo infinitamente lento dal mitico, cioè dal rappresentare inconscio, al pensiero logico, conforme al buonsenso ${ }^{94} »$. Gernet individua, pertanto, nella nozione mitica del valore un esempio particolarmente adatto a illustrare il modo di procedere della mentalità primitiva, che riguarda tutti i campi (economia, religione, politica, diritto, estetica), senza le distinzioni che interverranno con il pensiero logico: la nozione mitica del valore è dunque totale, come il carattere del sacro così descritto da Cassirer ${ }^{95}$ :

«Il senso e la potenza del "sacro" per il sentimento mitico primitivo non sono limitati ad alcun campo particolare, ad alcuna sfera singola dell'essere e ad alcuna sfera singola di valori. È piuttosto il complesso, la concrezione immediata e l'immediata totalità dell'esistenza ciò in cui questo senso si esprime.»

In Gernet, l'esempio del teras, espressione di una forza religiosa che trova nell'agalma la sua trasposizione mitica, si rivela particolarmente adatto a esemplificare il modo di procedere di un pensiero primitivo e religioso. La connessione profonda che intercorre tra il piano mitico e quello storico-religioso è ribadita nelle conclusioni: «En fin de compte, c'est bien l'idée de force religieuse qui peut être reconnue comme 
fondamentale dans la transposition mythique de l'agalma $a^{96}$.» Questa particolare forza religiosa di cui l'agalma è dotato gli è conferita, in ultima istanza, proprio dal suo legame con il sacro. Gli agalmata, tuttavia, non ricevono il loro valore dal fatto di trovare un impiego religioso, al contrario, essi devono il loro impiego religioso al valore di cui sono dotati. L'oggetto più ricco di implicazioni in questo senso è la larnax, che assurge nel mito a contenitore di oggetti di valore come il vello d'oro e racchiude, in qualche caso, l'eroe stesso. L'agalma possiede peraltro una virtù specifica, che è quella di esercitare un potere sociale, è questo un tratto che persiste nell'inconscio collettivo ben oltre l'età mitica. Questa virtù spiega perché il vello d'oro nel mito sia costantemente collegato alla figura del re, garante di prosperità e ricchezza per il popolo ${ }^{97}$.

L'agalma avrebbe dunque perso precocemente, già in età omerica, la sua polivalenza di significati in favore del suo valore strettamente economico, ma il pensiero mitico si è conservato laddove il significato economico dell'oggetto di valore ha prevalso ${ }^{98}$ :

«Ce qui peut laisser entendre que, dans la valeur et donc dans le signe même qui la représente, il y a un noyau irréductible à ce qu'on appelle vulgairement la pensée rationnelle.»

68 Proprio la persistenza di un pensiero mitico, che fa da «sfondo primitivo» al pensiero logico, testimonia dell'attualità dell'indagine di Gernet sulla nozione mitica del valore. Ma non è possibile comprendere tale attualità, se non si acquisisce piena consapevolezza della complessità di una simile indagine. L'ellenista francese risponde, infatti, con il rigore scientifico dovuto alla sua formazione di normalien, all'audace appello di Usener, che richiedeva alla mitologia di invadere il terreno di indagine della storia delle religioni, sul duplice versante delle rappresentazioni mitiche e delle azioni rituali. Gernet non si limita, tuttavia, ad accogliere l'auspicio espresso dal filologo tedesco; egli recepisce allo stesso tempo le istanze della neonata psicologia storica di Ignace Meyerson ispirata agli insegnamenti filosofici di Cassirer, che offrivano una chiave di interpretazione proprio di quella teoria della formazione dei concetti religiosi formulata da Usener. Solo considerando una simile apertura, per di più da parte di uno studioso che aveva meditato le istanze culturali di area tedesca e francese da una provvidenziale distanza accademica, si arriva ad apprezzare la solidità e la ricchezza di quel metodo nuovo per lo studio del mondo antico.

\section{NOTE}

1. Hermann Usener (Weilburg 1834 - Bonn 1905), figura guida della scuola filologica tedesca, la cosiddetta scuola di Bonn, è considerato a ragione uno dei padri fondatori della storia delle religioni. Tra le sue opere principali, oltre all'edizione degli Epicurea, Leipzig 1887 (tr. Epicurea : testi di Epicuro e testimonianze epicuree nella raccolta di Hermann Usener, traduzione e note di I. RAMELLI, presentazione di G. REALE, Milano 2002), ricordiamo i Götternamen. Versuch einer Lehre von der religiösen Begriffsbildung, Bonn 1896 (tr. I nomi degli dèi. Saggio di teoria della formazione dei concetti religiosi, introduzione e traduzione a cura di M. FERRANDO, revisione dei testi classici a cura di R.M. PARRINELLO, Brescia 2007), dove lo studioso indaga l'origine del monoteismo dal politeismo 
prendendo in esame gli dèi momentanei (Augenblicksgötter) e gli dèi particolari (Sondergötter) per arrivare agli dèi personali; le Sintfluthsagen, Bonn 1899 (tr. Le storie del diluvio, a cura di I. SFORZA, Brescia 2010), un'indagine comparata sui miti del diluvio nelle aree semitica, babilonese, indiana e greca; tra i numerosi contributi sulle radici pagane di leggende e santi cristiani, il saggio postumo Der heilige Tychon, Leipzig-Berlin 1907 (tr. San Ticone, a cura di I. SFORZA, Brescia 2007).

2. Louis-Jules Gernet (Parigi 1882 - 1962) si formò come grecista all'École Normale Supérieure sotto l'influsso della sociologia durkheimiana; insegnò filologia classica all'Università di Algeri dal 1921 al 1948 e poi sociologia giuridica dell'antichità greca presso l'École des Hautes Études a Parigi ove avviò gli studi di antropologia del mondo antico. I suoi contributi sulla scienza dell'antichità sono stati pubblicati ad opera di J.-P. VERNANT nella raccolta postuma Anthropologie de la Grèce antique, Paris 1968 (tr. Antropologia della Grecia antica, a cura di R. DI DONATO, Milano 1983) e nella successiva raccolta a cura di R. DI DONATO, con una prefazione di J.-P. VERNANT, Les Grecs sans miracle, Paris 1983 (tr. I Greci senza miracolo, Roma 1986). Alcuni testi frammentari sulla leggenda greca sono stati pubblicati di recente nel volume L. GERNET, Polyvalence des images. Testi e frammenti sulla leggenda greca, editi da A. SOLDANI, Pisa 2004.

3. Ignace Meyerson (Varsavia 1888 - 1983), medico e psicologo, condusse studi universitari in Francia. Fu direttore del Journal de Psychologie normale et pathologique dal 1938 fino alla morte e autore di una thèse dottorale che rimane la sua opera fondamentale, Les fonctions psychologiques et les oeuvres, Paris 1948 (tr. Psicologia storica : le funzioni psicologiche e le opere, prefazione di J.-P. VERNANT, Pisa 1989). Come osserva R. DI DONATO, «Immaginario e civiltà», prefazione a GERNET, Polyvalence, p. 9, il primato assoluto attribuito al referente durkheimiano dagli studiosi di area anglosassone a partire dal noto saggio di s.C. HUMPHREYS, «The Work of Louis Gernet», in History and Theory 10 (1971), p.172-196, tr. "L'opera di Louis Gernet", in EADEM (a cura di), Saggi antropologici sulla Grecia antica, prefazione di A. MOMIGLIANO, traduzione di P.P. Viazzo, Bologna 1979 (da cui si cita), p. 155-197, non attribuisce il rilievo necessario all'interlocuzione avuta già a partire dagli anni Trenta da Louis Gernet con Ignace Meyerson. Un tentativo di ricollocare la scuola sociologica francese in un milieu internazionale, attraverso la valorizzazione dei contatti avuti da alcuni suoi esponenti con la Völkerpsychologie tedesca, è stato condotto da R. DI DONATO, «Marcel Mauss et la Völkerpsychologie», in Revue européenne des sciences sociales, XXXIV, 105 (1996), p.67-74. Per l'influsso di Usener sulla messa a punto di un nuovo metodo di interpretazione del mito da parte di Gernet si farà qui riferimento alle considerazioni già espresse ne «Le storie del diluvio : percorsi di lettura», introduzione a USENER, Le storie del diluvio, p. 29-36.

4. La lettera fa parte del carteggio Gernet-Meyerson, conservato dallo psicologo insieme alla sua corrispondenza dal 1906 al 1983 e ora custodito nelle Archives Nationales a Fontainebleau (521 AP 1-65). I passaggi più significativi della lettera in questione sono stati pubblicati in italiano da $\mathrm{R}$. DI DONATO, Per una antropologia storica del mondo antico, Firenze 1990, p. 120-121, in francese da IDEM, «Immaginario e civiltà», p. 12.

5. «Per ventisette anni, dunque, dal 1920 al 1947, Gernet lavorò in relativo isolamento», ricorda HUMPHREYS, «The Work of Louis Gernet», p. 160. Sul progetto mai realizzato di un saggio sulla leggenda greca come documento di protostoria sociale cfr. DI DONATO, Per una antropologia, p. 119-130 e IDEM, «Immaginario e civiltà», p. 7-17.

6. L. GERNET, «La notion mythique de la valeur en Grèce», in Journal de psychologie 41 (1948), p. 415-462, rist. in GERNET, Anthropologie (da cui si cita), p. 93-137. La citazione è tratta dal fascicolo ALG II, 1 - $\Psi$ (Psychologie), 20r, GERNET, Polyvalence, p. 71.

7. E. CASSIRER, Philosophie der symbolischen Formen. Bd. I. Die Sprache; Bd. II. Das mythische Denken; Bd. III. Phänomenologie der Erkenntnis, Berlin 1923-1929, II, p. 140 (tr. di E. Arnaud, Filosofia delle forme simboliche, Firenze 1961-1966, p. 153).

8. GERNET, «Notion mythique», p. 99-100. 
9. USENER, Götternamen, p.V-VI (tr., p. 41-42) : «Der Stoff der Mythologie fällt also vollständig zusammen mit der inneren oder geistigen Vorgeschichte der Culturvölker, welche Mythologien geschaffen haben.»

10. USENER, Götternamen, p. VII (tr., p. 43) : «Nur durch hingebendes Versenken in diese Geistesspuren entschwundener Zeit, also durch philologische Arbeit vermögen wir uns zum Nachempfinden $\mathrm{zu}$ erziehen [...]. Reichere Beobachtung und Vergleichung gestattet weiter zu gehen, und wir erheben uns vom Einzelnen zum Ganzen, von den Erscheinungen zum Gesetz [...]. Je tiefer man gräbt, desto mehr wird man durch allgemeinere Erkenntnisse belohnt.» Cfr. E. CASSIRER, Sprache und Mythos : ein Beitrag zum Problem der Götternamen, Leipzig-Berlin 1925, p. 17-18 (tr. di V.E. Alfieri, Linguaggio e mito. Contributo al problema dei nomi degli dèi, Milano 1961, rist. 1976, p. 38-39).

11. USENER, Sintfluthsagen, cap. IV, p. 115-137 (tr., p. 127-144).

12. L. GERNET, «Frairies antiques», in Revue des Etudes Grecques 41 (1928), p. 313-359, rist. in GERNET, Anthropologie, p. 21-61, nota 24, p. 26.

13. Cfr. I. SFORZA, «Il miracolo della vite secondo Hermann Usener», introduzione a USENER, San Ticone, p. 5-28, in particolare 15-17.

14. GERNET, «Frairies antiques», p. 22-23. Gernet mostra inoltre di ritenere fondamentale la connessione cara a Usener tra mito e rito, quando afferma, a proposito di un testo di datazione incerta : «Ce texte aurait pu nous servir d'épigraphe : des thèmes rituels et des thèmes mythiques s'y peuvent raccorder, également élémentaires, mais déjà riches» (ibidem, p. 54).

15. Sul tema dei riti dionisiaci Gernet tornerà diversi anni più tardi in «Dionysos et la religion dionysiaque. Elements hérités et traits originaux», in Revue des Études Grecques 66 (1953), p. 229-247, rist. in GERNET, Anthropologie, p. 63-89.

16. GERNET, «Frairies antiques», p. 44-45.

17. Sul nesso linguaggio e mito in Gernet, vedi infra p. 147-149.

18. GERNET, «Frairies antiques», p. 45. Per lo «studio semantico» condotto programmaticamente da Gernet a partire dalle Recherches sur le développement de la pensée juridique et morale en Grèce. Étude sémantique, Paris 1917, vedi HUMPHREYS, «The Work of Louis Gernet», p. 174 e ss.

19. A. DIETERICH, Mutter Erde. Ein Versuch über Volksreligion, Leipzig 1905.

20. Cfr. GERNET, «Frairies antiques», nota 193, p. 58 : «On retiendra toutes les suggestions qui viennent, ici comme ailleurs, de M. Mauss, Essai sur le don». H. USENER, «Italische Mythen», in Rheinisches Museum XXX (1875), p. 182-229, rist. IDEM, Kleine Schriften, Leipzig-Berlin 1912-1914, IV, p. 93-143 è citato (p. 117 e ss.) alla nota 196, p. 58, a proposito dei riti di chiusura dell'anno, questa volta con un apprezzamento positivo; vedi anche nota 203, p. 60. Non è questa la sede per approfondire quali siano stati i modelli privilegiati, di area francese, seguiti da Gernet nel suo studio sulle leggende della Grecia antica. Sarà sufficiente ricordare che lo studioso francese adotta, in questa prima fase di ricerca sulla leggenda, la distinzione terminologica tra fable, conte, légende e mythe dell'antropologo A. VAN GENNEP, La formation des legendes, Paris 1910, per cui cfr. l'introduzione di R. DI DONATO a GERNET, Greci senza miracolo, p. 9-55, rist. in DI DONATO, Per una antropologia, p. 13-44, p. 25. Lo studio dei culti campestri in Grecia, oggetto del saggio «Frairies antiques», risente inoltre dell'influenza esercitata su Gernet dal sinologo Marcel Granet (1884-1940), che si era occupato della religione contadina in Cina : cfr. HUMPHREYs, «The Work of Louis Gernet», p. 165-167.

21. Ulrich von Wilamowitz-Möllendorff (Markowitz, Posnania 1848 - Charlottenburg, Berlino 1931) è stato uno dei più grandi maestri della filologia classica. Insegnò a Greifswald, Göttingen $e$ Berlino, fu socio straniero dell'Accademia dei Lincei. Tra le sue opere ricordiamo, oltre ai numerosi studi dedicati alla tragedia attica e alla lingua greca, le Homerische Untersuchungen, Berlin 1884, che segnano una tappa importante nell'approccio alla questione omerica, verso l'individuazione di due autori distinti dei poemi; la Geschichte der Philologie, Leipzig-Berlin 1921 e 
il citato Der Glaube der Hellenen, Berlin 1931-1932 dedicato alla religione greca. Di grande interesse il carteggio con Usener, Usener und Wilamowitz. Ein Briefwechsel 1870-1905, Leipzig-Berlin 1934 (2a ed. a cura di W.M. CALDER, Stuttgart-Leipzig 1994).

22. Revue de philologie, de littérature et d'histoire ancienne 60 (1934), p. 191-201, rist. in GERNET, Grecs sans miracle (da cui si cita), p. 104-115.

23. Ibidem, p. 105.

24. Ibidem, p. 106.

25. Sull'avversione di Wilamowitz per il primitivo, condivisa da alcuni studiosi inglesi suoi contemporanei come Gilbert Murray (1866-1957), si veda HUMPHREYS, «The Work of Louis Gernet», p. 162-163, la cui retrospettiva su Gernet prende le mosse proprio dalla «sua concezione dello sviluppo della società greca dei primordi», per cui cfr. ibidem, p. 164 e ss. L'evoluzione dell'approccio di Gernet al discusso tema delle «sopravvivenze» nelle leggende greche, dal tentativo di ricostruzione di una protostoria sociale ed economica all'indagine delle funzioni psicologiche sotto l'influsso dell'amico e allievo Ignace Meyerson è ben evidenziata da DI DONATO, Per una antropologia, p. 13-44.

26. GERNET, recensione a Der Glaube der Hellenen, p. 106.

27. Sull'allontanamento progressivo di Wilamowitz da Usener, del quale criticava l'adozione del metodo comparativo e l'interesse crescente per la storia delle religioni, si vedano le considerazioni e i rimandi bibliografici di A. WESSELS, Ursprungszauber. Zur Rezeption von Hermann Useners Lehre von der religiösen Begriffsbildung, Berlin 2003, soprattutto p. 7-8 e 73-74. Per l'influsso avuto dall'insegnamento di Usener sugli studi di storia delle religioni e di antropologia nelle università tedesche della prima metà del Novecento, cfr. R. SCHLESIER, "Arbeiter in Useners Weinberg". Anthropologie und antike Religionsgeschichte in Deutschland nach dem Ersten Weltkrieg, in $\mathrm{H}$. FLASHAR (a cura di), Altertumswissenschaft in den 20er Jahren. Neue Frage und Impulse, unter Mitarbeit von S. vOGT, Stuttgart 1995, p. 329-380.

28. Cfr. HUMPHREYS, «The Work of Louis Gernet», p. 186-187.

29. L. GERNET, «La cité future et le pays des morts», in Revue des Études Grecques 46 (1933), p. 293-310, rist. in GERNET, Anthropologie, p. 139-153, 145.

30. M.P. NILSSON, The Minoan-Mycenaean Religion and its Survival in Greek Religion, Lund 1927, p. 542 ss. : cfr. GERNET, «Cité future», p. 152-153. Di Nilsson Gernet aveva recensito in quel periodo The Mycenaean Origin of Greek Mythology, Berkeley 1932 per la Revue de philosophie, de littérature et d'histoire ancienne 59 (1933), p. 414-417, rist. GERNET, Grecs sans miracle, p. 99-104. Sul possibile peso che le critiche espresse da Gernet a Nilsson in tale occasione hanno avuto sulla fredda ricezione in area tedesca del saggio di L. GERNET, A. BOULANGER, Le génie grec dans la religion, Paris 1932, cfr. C. AMPOLO, «Fra religione e società», in Studi Storici 25, 1 (1984), p. 83-89. Nel già citato «Dionysos et la religion dionysiaque», p. 79, Gernet condivide l'omaggio reso a Nilsson da H. Jeanmaire, Dionysos. Histoire du culte de Bacchus, Paris 1951, «pour ce qui est de l'étendue du savoir, de la rigueur critique, de la sûreté de tout le travail de base». Un giudizio analogo esprimeva nei confronti di Nilsson l'eminente filologo Giorgio Pasquali per «la ricchezza e quasi completezza nella conoscenza delle fonti, assenza di ogni tendenziosità, ripugnanza a ipotesi troppo ardite», mentre «lo storico della religione greca più celebre, l'Usener, cedeva troppo spesso alle lusinghe del proprio ingegno, brillantissimo, ma proclive a generalizzazioni frettolose e a interpretazioni dei testi arbitrarie». Tanto che «di dottrine dell'Usener e del genero Dieterich il Nilsson fa spesso giustizia col silenzio» : prefazione di G. PASQUALI a M.P. NILSSON, Fondamenti di scienza delle religioni, Firenze 1950, rist. M.P. NILSSON, Le religioni degli antichi e i moderni, con una nota di G. PASQUALI, a cura di R. DI DONATO, Scandicci 1993, p. XX-XXI.

31. L. GERNET, «Dolon le loup», in Annuaire de l'Institut de Philologie et d'Histoire orientales et slaves 4 (1936), Mélanges Franz Cumont, p. 189-208, rist. in GERNET, Anthropologie, p. 154-171, nota 7, p. 155, 
dove Gernet fa riferimento al saggio di H. USENER, «Heilige Handlung», Archiv für Religionswissenschaft VII (1904), p. 281-339, rist. USENER, Kleine Schriften, IV, p. $422-467$ (p. 447 ss.).

32. La connessione postulata da Gernet tra il nome della città di Licorea, sulle pendici del Parnaso, e il racconto riportato da Pausania (X, 6, 2), secondo cui essa sarebbe stata fondata dagli uomini che durante il diluvio vi si rifugiarono spinti dall'ululato dei lupi, era stata rifiutata nelle Sintfluthsagen, p. 76-77 (tr., p. 98-99) da Usener, che preferiva ricondurre questo toponimo alla radice $\lambda u \kappa-$, da cui i numerosi epiteti di culto indagati nei Götternamen, tra i quali Zeus $\Lambda$ úкðıৎ annoverato anche da Gernet. Un certo apprezzamento per le intuizioni, qualche volta un po' azzardate, di Usener, è espresso da GERNET, «Dolon le loup», p. 162 : «La construction d'Usener est peut-être chancelante. Mais pour notre propos, il n'en faudrait pas tant» e nota 72, p. 166 : «Usener n'en a eu le sentiment».

33. Ibidem, p. 160.

34. Ibidem, p. 171.

35. Annales universitaires de l'Algérie, nouv. série 2 (1936), p. 14-29, rist. GERNET, Grecs sans miracle, p. 258-271, 258.

36. GERNET, «Notion mythique», p. 93. Un'analisi generale del saggio di Gernet è offerta dal contributo di S. VON REDEN, Re-evaluating Gernet : Value and Greek Myth, in R. BUXTON (a cura di), From Myth to Reason? Studies in the Development of Greek Though, Oxford 1999, p. 51-70. Per il lungo processo di riflessione sul mito che termina con la messa a punto del saggio sulla nozione mitica del valore, cfr. DI DONATO, «Immaginario e civiltà», p. 7-17.

37. Alla figura di Ignace Meyerson e alla sua opera è dedicata la seconda parte di DI DONATO, Per una antropologia, p. 133-205. Per l'apporto innovativo della psicologia storica di Meyerson si veda già M. DÉTIENNE, «Un renouvellement dans les sciences humaines : la psychologie historique et comparative d'Ignace Meyerson», in Synthèse 184 (1961), p. 3-11. Il volume di J.-P. VERNANT, Mythe et pensée chez les Grecs, che porta il sottotitolo Études de psychologie historique, Paris 1965, costituisce, come appare chiaro fin dall'introduzione, la più significativa ripresa della fruttuosa interlocuzione tra Gernet e Meyerson.

38. La lettera, pubblicata in italiano da DI DONATO, Per una antropologia, p. 129 e in francese in IDEM, «Immaginario e civiltà», p. 13, è considerata dallo stesso come termine cronologico di quel processo di riflessione che avrebbe portato l'ellenista a rinunciare al suo precedente progetto di un essai général d'analyse du mythe pour l'intelligence de la préhistoire sociale da lui annunciato allo stesso Meyerson nel 1942, in favore dell'elaborazione più circoscritta e matura del saggio sulla nozione mitica del valore.

39. E. CASSIRER, «Le langage et la construction du monde des objets», in Journal de psychologie 30 (1933), p. 18-44, rist. J.-C. PARIENTE (a cura di), Essais sur le langage, Paris 1969, p. 37-68, tr. di G. Mininni, Il linguaggio, Bari 1976, p. 55-84.

40. All'indagine di Gernet sulla nozione mitica del valore si rifà espressamente M. DETIENNE ne Les maîtres de vérité dans la Grèce archaïque, Paris 1967, dove l'autore si propone di indagare il significato «pre-razionale» della nozione greca di Alétheia e di definire il cambiamento che tale significato subisce nel pensiero razionale. Il saggio si configura come una ripresa e uno sviluppo dell'articolo «La notion mythique d'A $\lambda \hat{n} \theta \varepsilon ı \alpha »$, Revue des Études Grecques 73 (1960), p. 27-35, nato dall'interlocuzione di Detienne con Gernet, come chiarito nel primo capitolo del saggio, "Vérité et Société", nota 12, p. 8.

41. GERNET, «Notion mythique», p. 93-94.

42. Ibidem, p. 94.

43. Ivi, nota 3.

44. Sulle forme arcaiche di scambio, a partire dal contributo di Gernet, vedi N.F. PARISE, «Rilievi inattuali su "segni premonetari" e nascita della moneta in Grecia», in Studi Storici 25, 1 (1984), p. 55-58. Verte essenzialmente sul valore «economico» dell'agalma il contributo di VON REDEN, Re- 
evaluating Gernet, a cui si rinvia per la bibliografia di area anglosassone su questo tema. Per i significati del dono nell'epica greca arcaica, si veda la dettagliata analisi di E. SCHEID-TISSINIER, Les usages du don chez Homère : vocabulaire et pratiques, Nancy 1994.

45. GERNET, «Notion mythique», p. 99.

46. CASSIRER, Sprache, p. 8 (tr., p. 20) : «Sie werden nicht aus einer fertigen Welt des Seins herausgehoben, sie sind keine blossen Gebilde der Phantasie, die sich aus der feststehenden empirisch-realen Wirklichkeit der Dinge ablösen und sich über sie wie ein leichter Nebel erheben, sondern sie stellen für das primitive Bewusstsein das Ganze des Seins dar». GERNET, «Notion mythique», nota 15, p. 99 spiega così la scelta del termine : «On ne chicanera pas sur le mot : même si on qualifie plus spécialement de mythes un certain ordre de récits, différent de celui qui nous occupe, il y a un même mode de représentation, avec des thèmes parfois communs aux différents étages d'invention que désignent les termes consacrés de mythe, conte, légende. Et au point de vue psychologique, il n'y a que cela qu'on ait à retenir, ici du moins.»

47. GERNET, «Notion mythique», p. 99.

48. MEYERSON, Fonctions psychologiques, p. 31-71.

49. Si veda soprattutto CASSIRER, Philosophie, II, «Zweiter Abschnitt. Der Mythos als Anschauungsform. Aufbau und Gliederung der räumlich-zeitlichen Welt im mythischen Bewusstsein», p. 93 ss. (tr. "Seconda parte. Il mito come forma di intuizione. Struttura e articolazione del mondo spazio-temporale nella coscienza mitica», p. 107 ss.). Un influsso di Cassirer su Meyerson è ipotizzato da DI DONATO, «Immaginario e civiltà», p. 15, che vi accenna anche in IDEM, «Marcel Mauss», p. 74. La possibilità di una conoscenza diretta di Cassirer da parte di Gernet, che era senza dubbio un attento lettore di Usener, è inoltre ammessa nel paragrafo dedicato alle «métamorphoses de la pensée mythique» da M. DETIENNE, Les Grecs et nous. Une anthropologie comparée de la Grèce ancienne, Paris 2005, p. 106 : «Pour l'heure, la question est celle de la "pensée mythique", si présente dans l'enquête ouverte en 1960. Présente à travers Louis Gernet, qui, ici ou là, faisait référence à des intuitions de H. Usener, mais qui en parlait avec la conviction d'un disciple de Durkheim, peut-être aussi d'un lecteur de Cassirer, qui avait, lui, consacré un volume entier...». Al di là di questi sporadici riferimenti, la questione del duplice influsso di area tedesca, Usener e Cassirer, sullo studio del pensiero mitico da parte di Gernet, non è stata, a quanto mi risulta, oggetto di approfondimento.

50. Cfr. la prefazione di J.-P. VERNANT a MEYERSON, Psicologia storica, p. 11.

51. MEYERSON, Fonctions psychologiques, p. 37-38.

52. Ibidem, p. 40-49; cfr. p. 41 e nota 1, a proposito del mana : «Cassirer caractérise de manière très heureuse ces côtés non matériels du mana en disant qu'il est la catégorie de l'exceptionnel, non tel contenu spécial rare, mais l'exceptionnel même.»

53. «Esquisse d'une théorie générale de la magie», Année Sociologique 7 (1902/1903), p. 1-146, rist. in M. Mauss, Sociologie et Anthropologie, Paris 1950, p. 3-141. Cfr. CASSIRER, Philosophie, II, p. 98-99, nota 3 (tr., p. 111, nota 2).

54. CASSIRER, Sprache, p. 2 (tr., p. 11) : «Dass Name und Wesen in einem innerlich-notwendigen Verhältnis zueinander stehen, dass der Name das Wesen nicht nur bezeichnet, sondern dass er das Wesen selbst ist und dass die Kraft des Wesens in ihm beschlossen liegt: dies gehört zu den Grundvoraussetzungen der mythischen Anschauung selbst.»

55. Ibidem, p. 3 (tr., p. 12) : «[...] die noch unverkennbar an die alten Methoden der griechischen Sophistik erinnern.»

56. Ibidem, p. 3-4 (tr. p. 12-14). Cfr. DETIENNE, Les Grecs et nous, soprattutto il secondo capitolo «Du mythe à la mythologie entre Américains et Grecs», p. 27-61. Sulla nascita di una «science des mythes» si veda inoltre il contributo dello stesso autore L'invention de la mythologie, Paris 1981.

57. CASSIRER, Sprache, p. 12 (tr., p. 27) : «Mythologie ist die Lehre ( $\lambda$ óyoৎ) vom Mythos oder die Formenlehre der religiösen Vorstellungen.» 
58. Ibidem, p. 17-18 (tr., p. 38-39) : «Die Betrachtung sucht bis zu einem Punkte vorzudringen, an dem Beides, der Gott wie sein Name, im Bewusstsein zuerst entspringt. Dieses "Entspringen" [...] wird aus der Grundstruktur des sprachlichen und mythischen Bewusstseins schlechthin, aus einem allgemeinen Gesetz der sprachlichen und religiösen Begriffsbildung $\mathrm{zu}$ verstehen versucht. Hier stehen wir daher nicht mehr auf dem Boden der Geschichte, sondern auf dem der Phänomenologie des Geistes».

59. Cfr. GERNET, «Notion mythique», p. 99 e USENER, Götternamen, p. V-VI (tr., p. 41-42, corsivo mio) : «Es sind die Kindheitsstufen der Völker, auf denen sich Volksthümlicher Glaube bildet und zu mythischen Formen gestaltet; alles was in dieser Zeit das Gemüth des Volkes erregt, die ganze Aussenwelt, die ersten Regungen des Bewusstseins [...] das alles lebt als Niederschlag im Mythenschatze des Volkes fort; denn jedes neue und unbekannte tritt zunächst als ein göttliches Wesen an jene Menschen heran. Der Stoff der Mythologie fällt also vollständig zusammen mit der inneren oder geistigen Vorgeschichte der Culturvölker, welche Mythologien geschaffen haben».

60. CASSIRER, Philosophie, II, p. 93-96 (tr., p. 108) : «Diese Bewusstsein ist und lebt im unmittelbaren Eindruck, dem es sich überlässt, ohne ihn an einem anderen zu "messen". Der Eindruck ist ihm kein bloss Relatives, sondern ein Absolutes; er ist nicht "durch" etwas anderes und hängt nicht von einem andern, als seiner Bedingung, ab; sondern er bezeugt und bewährt sich durch die einfache Intensität seines Daseins; durch den unwiderstehlichen Zwang, mit dem er sich dem Bewusstsein aufdrängt.»

61. GERNET, Polyvalence, p. 77. La citazione è da H. USENER, «Mythologie», Archiv für Religionswissenschaft VII (1904), p. 6-32, rist. in H. USENER, Vorträge und Aufsätze, Leipzig 1907, p. 37-65, in particolare 57-58 (tr. USENER, San Ticone, p. 167).

62. GERNET, Polyvalence, p. 40. Il riferimento è a USENER, «Mythologie», p. 39 (tr., p. 155): «Dass die griechische Mythologie, wie sie in den vorzüglichen Handbüchern betrieben wird, mit denen wir seit 1854 versehen worden sind, nicht Wissenschaft heissen könne, wäre überflüssig, förmlich zu beweisen, so viele auch heute noch des entgegengesetzten Glaubens leben. Sie löst eine schwere und verwickelte Aufgabe, indem sie die Gestalten der Götter und Heroen, wie sie im Kultus, in der Dichtung und bildenden Kunst hervortreten, wieder vor uns aufrichtet und den verschlungenen Wegen der Mythographie nachgeht, um Wachstum und Umbildung der Sagen zu ermitteln. Aber über diese vorbereitende Tätigkeit hinauszugehen, dazu fehlen ihr die Mittel und Voraussetzungen.»

63. GERNET, Polyvalence, p. 42.

64. Ibidem, p. 48.

65. Ibidem, p. 49.

66. Ibidem, p. 71.

67. Ibidem, p. 72.

68. GERNET, «Notion mythique», p. 100.

69. F. DE SAUSSURE, Cours de linguistique générale, publié par CH. BALly e A. SECHEHAYE avec la collaboration de A. RIEDLINGER, Paris 1931, p. 158-162 (tr. Corso di linguistica generale. Introduzione, traduzione e commento di T. DE MAURO, Bari 1967).

70. Ibidem, p. 176.

71. Ibidem, p. 177.

72. Ibidem, p. 179.

73. GERNET, «Notion mythique», p. 101 : «L'objet lui même a une valeur singulière qui s'apparente à la valeur religieuse : finalement [...] le trépied est consacré à un dieu.»

74. CASSIRER, Philosophie, II, p. 97 (tr., p. 109) : «Immer ist es dieser eigentümliche Zug zur "Transzendenz", der alle Inhalte des mythischen und des religiösen Bewusstseins miteinander verknüpft. Sie alle enthalten in ihrem blossen Dasein und in ihrer unmittelbaren Beschaffenheit eine Offenbarung, die doch eben als solche noch die Art des Geheimnisses behält - und 
ebendieses Ineinander, diese Offenbarung, die zugleich Enthüllung und Verhüllung ist, prägt dem mythisch-religiösen Inhalt seinen Grundzug, prägt ihm den Charakter der "Heiligkeit" auf.» 75. GERNET, «Notion mythique», nota 22, p. 102. Si veda in particolare il capitolo terzo di USENER, Sintfluthsagen, «Das Götterknäblein in der Truhe», p. 80-114 (tr., p. 101-125).

76. GERNET, «Notion mythique», p. 103-104.

77. Ibidem, p. 104.

78. Ibidem, p. 108.

79. Ibidem, p. 99-100.

80. Ibidem, p. 110.

81. Ibidem, p. 113.

82. Ibidem, p. 116.

83. Ibidem, p. 116-119.

84. Ibidem, p. 119. Corsivo mio.

85. Cfr. SFORZA, «Storie del diluvio», p. 29-36.

86. USENER, Sintfluthsagen, p. 181-182 (corsivo mio, tr. p. 179) : «Das Sagengewebe zu entwirren, an welchem so viele Geschlechter von Dichtern, Logographen und systematisierenden Mythographen gearbeitet haben, würde überaus schwierig oder vielmehr unmöglich sein, wenn es nicht in so vielen Fällen einfachere, alterthümlichere, von dem Umbildungstriebe der Dichtung unberührte Gestaltungen des Mythos gäbe, die sich zur Vergleichung darbieten und den Kern der entwickelten, dh. von der Dichtung überwucherten Sage zu erkennen gestatten.»

87. GERNET, «Notion mythique», p. 124. Corsivo mio.

88. USENER, Sintfluthsagen, p. 184 (tr., p. 181) : «Vereinigt hat man die beiden Bilder des Viehs und des Goldes in einem dritten, dem goldenen Widder der Pelopiden, des Phrixos, des Mandrobulos.» 89. GERNET, «Notion mythique», p. 125.

90. Ibidem, p. 129.

91. Ibidem, p. 131. Si vedano in proposito le considerazioni espresse in SFORZA, «Il miracolo della vite», p. 7-11.

92. USENER, «Mythologie», p. 40 (tr., p. 156) : «Nur wenn sie in die Geschichte der Religion eines Volkes einbezogen ist, vermag dessen Mythologie wissenschaftliche Bearbeitung zu finden.»

93. Ibidem, p. 43 (tr., p. 157) : «Man darf sagen, dass der ursprüngliche Mensch nur religiös apperzipiert.»

94. Ibidem, p. 46 (tr., p. 160) : «Der menschliche Geist arbeitet sich unendlich langsam aus mythischem, d. h. unbewusstem Vorstellen hindurch zu vernunftgemässem logischen Denken.»

95. CASSIRER, Philosophie, II, p. 97-98 (tr., p. 109) : «Der Sinn und die Macht des "Heiligen" ist für das ursprüngliche mythische Gefühl auf keinen Sonderbezirk, auf keine einzelne Seinssphäre und auf keine einzelne Wertsphäre beschränkt. Vielmehr ist es die ganze Fülle, die unmittelbare Konkretion und die unmittelbare Totalität des Daseins und Geschehens, woran dieser Sinn sich ausprägt.»

96. GERNET, «Notion mythique», p. 132.

97. Ibidem, p. 133-134.

98. Ibidem, p. 137. L'interesse di Gernet per l'etica del dono nelle società arcaiche, indagata già da M. MAUSS, «Essai sur le don. Forme et raison de l'échange dans les sociétés archaïques», Année Sociologique, nouv. série 1 (1925), p. 30-186 (tr. Saggio sul dono. Forme e motivo dello scambio nelle società arcaiche. Introduzione di M. Aime, Torino 1965), si concretizza in tre contributi strettamente correlati : il primo, in ordine di tempo, è «Jeux et droit. Remarques sur le XXIII chant de l'Iliade», Comptes rendus de l'académie des Inscr. et Belles Lettres 1947, p. 572-574 (rist. in Droit et société dans la Grèce ancienne, Paris 1955, p. 9-18); il secondo «La notion mythique de la valeur» (1948) e il terzo «Droit et prédroit en Grèce ancienne», Année Sociologique, III série (1948-1949), 1951, p. 21-119 (rist. GERNET, Anthropologie, p. 171-260), pubblicato significativamente 
nello stesso numero dell'Année Sociologique in cui compare il saggio di E. BENVENISTE, Don et échange dans le vocabulaire indo-européen, p. 11-20. Le osservazioni di Gernet hanno variamente ispirato il volume collettivo Problèmes de la guerre en Grèce ancienne, sous la direction de J.-P. VERNANT, Paris 1968, nella cui introduzione Vernant mette in luce la complementarità tra guerra e matrimonio, nonché tra guerra e giochi, «deux faces... d'un même phénomène social». Sulle orme di GERNET, Droit et société, si sviluppano inoltre le riflessioni di DETIENNE, Maitres de vérité, p. 81-103 sui giochi funerari come «terrain de prédroit». La più ampia disamina, di poco successiva, sul tema del dono di SCHEID-TISSINIER, Usages $d u$ don è stata ripresa di recente da R. DI DONATO, Aristeuein. Premesse antropologiche ad Omero, Pisa 2006, p. 25-33. Sulla centralità del bottino in alternativa al corpo di un caduto nelle raffigurazioni di duello epico, si veda infine I. SFORZA, L'eroe e il suo doppio. Uno studio linguistico e iconologico, prefazione di F. LISSARRAGUE, Pisa 2007, p. 111-119.

\section{RIASSUNTI}

L'influence d'Hermann Usener (1834-1905), philologue allemand et historien des religions, sur l'helléniste français Louis Gernet (1882-1962) n'a pas encore fait l'objet d'une étude approfondie. Dans les notes de Gernet sur les rapports entre mythologie et langage, datables environ des années Quarante du siècle dernier, on retrouve un grand nombre de citations d'Usener. Ces considérations conduisent Gernet à déterminer, dans La notion mythique de la valeur en Grèce (1948), les connexions entre plusieurs histoires légendaires et à l'intérieur d'une même histoire. Dans ce texte il développe des idées révolutionnaires suggérées par Usener dans les Sintfluthsagen (1899) et dans Mythologie (1904) grâce à l'apport de la psychologie historique. Ignace Meyerson (1888-1983), fondateur de cette nouvelle discipline, avait précisé sa pensée, à son tour, sous l'influence du philosophe allemand Ernst Cassirer (1874-1945), qui avait commenté, dans Sprache und Mythos (1925), Die Götternamen (1896) d'Usener. C'est à travers cette triangulation exceptionnelle que l'on peut remonter aux origines de l'anthropologie historique en France.

The influence of Hermann Usener (1834-1905), a German philologist and historian of religions, over the French hellenist Louis Gernet (1882-1962) has not as yet been the object of a thoroughgoing study. In Gernet's notes on the relationships between mythology and language, dating approximately from the 1940's, a great many quotations from Usener are to be found. Such considerations led Gernet to sketch out in La notion mystique de la valeur en Grèce the connections between several legendary stories and within the same story. In that text he develops revolutionary ideas suggested by Usener in the Sintfluthsagen (1899) and in Mythologie (1904) thanks to the contribution of historical psychology. Ignace Meyerson (1888-1983) the founder of that discipline had in his turn clarified his thought under the influence of Ernst Cassirer (1874-1945) the German philosopher who had in Sprache und Mythos (1925) commented upon Usener's Die Götternamen (1896). It is through that exceptional triangulation that we can go back to the origins of historical anthropology in France. 
INDICE

Mots-clés : anthropologie historique, histoire de la culture, légende, littérature grecque, mythologie grecque, notion de la valeur, psychologie historique, représentation mythique, richesse

Keywords : historical anthropology, history of culture, legend, Greek literature, Greek mythology, notion of value, historical psychology, mythical representation, wealth

\section{AUTORE}

ILARIA SFORZA

Roma (Italia)

kakridis@hotmail.com 